\title{
Relación del desarrollo del color con el contenido de antocianinas y clorofila en diferentes grados de madurez de mortiño (Vaccinium floribundum)
}

\section{(Development relationship of color with anthocyanins and chlorophyll content in diferent degrees of maturity of mortiño (Vaccinium floribundum))}

\author{
Mónica Arteaga Dalgo ${ }^{1}$, Maria Jose Andrade Cuvi ${ }^{1}$, Carlota Moreno Guerrero ${ }^{1}$
}

\begin{abstract}
Resumen:
Se estudió la relación entre el desarrollo del color con el contenido de antocianinas y clorofila en diferentes grados de madurez de mortiño (Vaccinum floribundum). Los frutos se cosecharon según su color superficial de $100 \%$ verde hasta 100\% negro. Se midió el color según la escala $\mathrm{CIE} \mathrm{L*a*b*} \mathrm{y} \mathrm{se} \mathrm{determinó} \mathrm{la} \mathrm{diferencia} \mathrm{de} \mathrm{color}(\Delta E)$. El contenido de antocianinas y clorofila se realizó por espectrofotometría UV-VIS. Se establecieron cinco estados de madurez: (1) verde, (2) verde/rosado, (3) rosado, (4) rosado/negro y (5) negro. Se encontró que la maduración presenta una relación inversa con la luminosidad y la saturación de color. Se obtuvieron valores positivos de $a^{*}$ y negativos de $b^{*}$ para los estados de madurez 3,4 y 5 (color rojo y azul); y valores negativos de $\mathrm{a}^{*}$ y positivos de $\mathrm{b}^{*}$ para los estados 1 y 2 (color verde y amarillo). Entre los estados 4 y 5 se encontró la menor diferencia de color $(\Delta \mathrm{E})$. Entre los estados 1 y 5 se observó una disminución de $0,052 \mathrm{mg} / \mathrm{g}$ de clorofila y un incremento de $13700 \mathrm{mg} / \mathrm{kg}$ del contenido de antocianinas. Se encontró una relación directa entre el tono del color (Hue) y el contenido de compuestos con actividad antioxidante constituyendo un antecedente para futuras investigaciones y por otro lado, el aprovechamiento de este fruto en diferentes estados de madurez.
\end{abstract}

Palabras clave: Mortiño, antocianinas, clorofila, color, madurez

\begin{abstract}
:
The relationship between color development with anthocyanins and chlorophyll content at different stages of maturity of mortiño (Vaccinum floribundum) was studied. The fruits were harvested by surface color from $100 \%$ green to $100 \%$ black. The color scale was measured according to $\operatorname{CIE~} L^{*} a^{*} b^{*}$ and color difference $(\Delta E)$ was determined. The anthocyanin and chlorophyll content was performed by UV-VIS spectrophotometry. The following scale was used to establish the stage of maturity: (1) green, (2) green/pink, (3) pink, (4) pink/black and (5) black; It was observed that increasing the maturation stage, the brightness and color saturation decrease. Results showed positive values of $a^{*}$ and negative of $b^{*}$ for maturity stages 3,4 and 5 (red and blue); and negative values of $a{ }^{*}$ and positive of $b{ }^{*}$ for states 1 and 2 (green and yellow). Smallest differences in color $(\Delta \mathrm{E})$ were found between states 4 and 5 . Among the states 1 and 5 was evident a decreased of $0,052 \mathrm{mg} / \mathrm{g}$ in chlorophyll content and an increase of $13700 \mathrm{mg} / \mathrm{kg}$ in anthocyanins. The degree of maturity of the fruit has a direct relationship between the color tone and content of components with antioxidant activity constituting a precedent for future researches and its use of this fruit in different stages of maturity.
\end{abstract}

Keywords: Mortiño, anthocyanins, chlorophyll, color, maturity.

\footnotetext{
${ }^{1}$ Universidad Tecnológica Equinoccial, Facultad Ciencias de la Ingeniería, Centro de Investigación de
} Alimentos CIAL, Quito - Ecuador (acmj2221@ute.edu.ec) 


\section{Introducción}

El mortiño (Vaccinum floribumdum) es un fruto climatérico perteneciente a la familia Ericaceae, conocido como "Andean Blueberry", es una especie endémica que se desarrolla de forma silvestre en los páramos ecuatorianos entre los 2800 y 4000 m.s.n.m. (Jimenez, 2004; Popeneo, King, León, \& Sumar, 1989; Dávila, Andrés, Stalin, \& Francisco, 2010; Pérez \& Valdiviezo, 2007).

Durante el proceso de maduración el mortiño sufre una serie de cambios de color: desde verde cuando aún no madura, rosado cuando alcanza la madurez fisiológica hasta alcanzar un color negruzco cuando llega a la madurez hortícola o comercial (Vargas, 2002; Patiño, 2002).

El color es una propiedad de la materia que se relaciona directamente con el espectro de luz y se puede medir físicamente debido a su energía radiante o intensidad, así como también por su longitud de onda. El cambio de coloración que acompaña a la madurez fisiológica de las frutas se emplea comúnmente para determinar el índice de madurez de las mismas. A pesar de que el ojo humano es extremadamente sensible a las diferencias entre colores, sólo es capaz de dar una medición subjetiva, es por esta razón que se utilizan aparatos precisos que combinan la electrónica y la óptica permitiendo realizar medidas objetivas del color (Badui, 1981).

Las frutas tienen una alta concentración de pigmentos (la mayoría de ellos con actividad antioxidante) fácilmente identificables por colores característicos presentes en su parte externa. Es el caso de las antocianinas que poseen colores azul, púrpura, violeta, magenta, rojo y naranja. Estos compuestos están ampliamente distribuidos en la naturaleza tanto en flores y frutas como en raíces y hojas, presentan una reconocida capacidad antioxidante que ha sido ampliamente estudiada; por otro lado, las clorofilas se caracterizan por el color verde e intervienen en la fotosíntesis en las plantas, algas y bacterias fotosintéticas (Tupuna, 2012; Pokorny, Yanishlieva, \& Gordon, 2001; Skoog, West, Holler, \& Crouch, 2001; Fennema, 2000).

Existe una relación entre el color y la maduración de las frutas que se refleja al momento de la cosecha, postcosecha y comercialización, debido a los cambios de pigmentación que tienen lugar durante el desarrollo y maduración del producto sobre la planta. De este modo se puede relacionar el contenido antioxidante con el color de las frutas, pudiendo ser medido gracias a la propiedad que posee cada pigmento para absorber una cierta longitud de onda del espectro visible (Badui, 1981; Sinha, Sidhu, Barta, Wu, \& Cano, 2012). El objetivo del presente trabajo de investigación fue relacionar el desarrollo del color con el contenido de antocianinas y clorofila en diferentes grados de madurez del mortiño. 


\section{Metodología}

\subsection{Material vegetal y medida de color superficial}

Frutos de mortiño en diferentes grados de madurez fueron cosechados en el páramo "El Pedregal", ubicado en el cantón Mejía, Provincia de Pichincha. Inmediatamente se trasladaron al Laboratorio de Biotecnología (Facultad Ciencias de la Ingeniería-UTE) donde se clasificaron según su color superficial (relacionado con su grado de madurez). Se realizó la medición del color usando un colorímetro Konica Minolta CR 400 (escala CIE $L^{*}, a^{*}, b^{*}$ ); se obtuvo valores de $L^{*}$ para luminosidad comprendidos entre 0 y 100 ; valores de $a^{*}$ para colores rojos (positivos) y verdes (negativos), y valores de $b^{*}$ para colores azules (negativos) y amarillos (positivos) (Konica Minolta, 2012; Zheng, Chien, Shiow, \& Wei, 2003).

Además se determinaron los parámetros de color: Chroma - $\mathrm{C}^{*}$ (indicador de intensidad 0 saturación de color) y el ángulo Hue - H (tono, matiz o longitud de onda dominante), para lo cual se utilizó los valores de $a^{*} y b^{*}$, según las ecuaciones 1 y 2 .

$$
\begin{gathered}
C^{*}=\left[\left(a^{*}\right)^{2}+\left(b^{*}\right)^{2}\right]^{1 / 2} \\
\mathrm{H}=\operatorname{Tan}^{-1}\left(\frac{\mathrm{a} *}{\mathrm{~b} *}\right)
\end{gathered}
$$

Mediante la ecuación 3 se determinó la diferencia de color $(\Delta \mathrm{E})$ entre los diferentes estados de madurez, en términos de Luminosidad, $a^{*} \mathrm{y} b^{*}$.

$$
\Delta \mathrm{E}^{*} a b=\left[\left(\Delta L^{*}\right)^{2}+\left(\Delta a^{*}\right)^{2}+\left(\Delta b^{*}\right)^{2}\right]^{1 / 2}
$$

\subsection{Extracción y cuantificación de antocianinas}

Se cuantificó el contenido de antocianinas según el método de Beas (2011) con ligeras modificaciones. Se pesó entre 0,02 y 5 gramos (según el estado de madurez) de tejido congelado y triturado, se agregó $10 \mathrm{ml}$ de $\mathrm{HCl}$ 1\% en metanol, se agitó por 15 min y se centrifugó a 6000 rpm durante $5 \mathrm{~min}$, posteriormente se retiró el sobrenadante y se conservó protegido de la luz; al pellet se añadió $10 \mathrm{~mL}$ de solvente, se agitó y centrifugó nuevamente. Se midió la absorbancia del sobrenadante a 540 $\mathrm{nm}$ y se expresó el contenido de antocianinas totales en $\mathrm{mg} / \mathrm{kg}$ (como equivalentes de cianidina-3-glucósido), según la ecuación 4.

$$
C=\left(\frac{A}{\varepsilon}\right)\left(\frac{V o l}{1000}\right)(P M)\left(\frac{1}{\text { peso muestra }}\right)\left(10^{6}\right)
$$

Donde,

$\mathrm{C}=$ Concentración de Antocianinas $(\mathrm{mg} / \mathrm{kg})$

A = Absorbancia máxima

$\varepsilon=$ Absortividad actividad molar cianidina-3-glicósido $(25955 / \mathrm{cmM})$

Vol= Volumen total de extracto

$\mathrm{PM}=$ Peso molecular cianidina-3-glicósido (449Da) 


\subsection{Extracción y cuantificación de clorofila}

Se pesaron de 0,05 a 5 gramos de tejido congelado y triturado (según el estado de madurez de la fruta), a los cuales se añadió $15 \mathrm{ml}$ de acetona al $80 \%$. El homogenizado resultante fue centrifugado a $12000 \mathrm{rpm}$ durante dos minutos. Para la medición de contenido de clorofila se utilizó el método de Arnon (1949), en el cual se midió la absorbancia de la muestra a 645 y 662 $\mathrm{nm}$. Se determinó el contenido de clorofila a (ecuación 5), clorofila $\beta$ (ecuación 6) y clorofila total (ecuación 7).

$$
\begin{aligned}
& \text { Clorofila } \alpha\left(\frac{\mathrm{mg}}{\mathrm{g}}\right)=\frac{\left[\left(12,7 \times \mathrm{A}_{663}\right)-\left(2,6 \times \mathrm{A}_{645}\right)\right] \times \mathrm{mL} \text { Acetona }}{\mathrm{mg} \mathrm{de} \mathrm{muestra}} \\
& \text { Clorofila } \beta\left(\frac{\mathrm{mg}}{\mathrm{g}}\right)=\frac{\left[\left(12,7 \times \mathrm{A}_{645}\right)-\left(2,6 \times \mathrm{A}_{663}\right)\right] \times \mathrm{mL} \text { Acetona }}{\mathrm{mg} \text { de muestra }}
\end{aligned}
$$

Clorofila Total $=$ Clorofila $\alpha+$ Clorofila $\beta$

\subsection{Análisis estadístico y diseño experimental}

Se utilizó el diseño experimental completamente al azar. Se realizó el análisis de varianza (Anova simple) y las medias se compararon por el test de Tukey con un nivel de significancia de 0,05, se empleó el programa Statgraphics Centurion XV.

\section{Resultados}

\subsection{Medida de color superficial}

Los frutos fueron cosechados en diferentes estados de madurez y se clasificaron según su color superficial utilizando la escala que se observa en la tabla 1.

Tabla 1. Clasificación del mortiño por grado de madurez

\begin{tabular}{|c|c|}
\hline Estado de Madurez & Característica \\
\hline 1 & $\begin{array}{c}\text { Color verde } 100 \% \\
\text { rosado } 50 \% \text { verde }-50 \%\end{array}$ \\
\hline 2 & Color rosado $100 \%$ \\
\hline 4 & Color $50 \%$ negro $-50 \%$ rosado \\
\hline 5 & Color negro $100 \%$ \\
\hline
\end{tabular}


En la tabla 2 se presentan los resultados de los parámetros de color de los diferentes grados de madurez de mortiño. Se observó un descenso de los valores de $L$ a lo largo de la maduración. Los frutos de color verde $100 \%$ (estado de madurez 1) presentaron un valor de luminosidad de 37 que se mantuvo prácticamente constante en los frutos hasta el estado de madurez 2 (color verderosado), mientras que al alcanzar la madurez fisiológica en el estado de madurez 3 (color rosado) el valor de luminosidad disminuyó a 30,92; valor similar al encontrado en el estado de madurez 4 (color negro-rosado); cuando los frutos alcanzaron la madurez hortícola en el grado de madurez 5 (color negro), se determinó un valor de luminosidad de 28. Zheng, Chien, Shiow y Wei (2003) reportaron para arándano maduro valores de $\mathrm{L}=32$ (mayor al obtenido para mortiño), probablemente esta diferencia se deba a la coloración que caracteriza al arándano cuando alcanza su madurez comercial (color azul). Por lo contrario, en productos donde el cambio de color durante la maduración es de amarillo a verde como la soya se registran incrementos de los valores de L (Sinnecker, Gomes, Areas, \& Marquez, 2002). Cuando el color se acerca a blanco los valores de luminosidad son mayores que cuando el color tiende a negro (Kónica Minolta, 2012).

Tabla 2. Medida de parámetros de color $\mathrm{L}, \mathrm{a}^{*}, \mathrm{~b}^{*}, \mathrm{C}^{*}$ y $\mathrm{H}$ para los diferentes estados de madurez del mortiño (Vaccinium floribundum)

\begin{tabular}{|c|c|c|c|c|c|}
\hline \multirow{2}{*}{$\begin{array}{l}\text { Estado de } \\
\text { madurez }\end{array}$} & \multicolumn{5}{|c|}{ Parámetros de color } \\
\hline & $\mathbf{L}$ & $\mathbf{a}^{*}$ & $\mathbf{b}^{*}$ & $\mathbf{C}^{*}$ & $\mathbf{H}$ \\
\hline 1 & $37.36^{a}$ & $-5.68^{f}$ & $10.37^{a}$ & $11.84^{\mathrm{a}}$ & $155.46^{\mathrm{C}}$ \\
\hline 2 & $37.74^{\mathrm{b}}$ & $-0.51^{d}$ & $6.38^{\mathrm{b}}$ & $6.53^{\mathrm{b}}$ & $204.04^{b}$ \\
\hline 3 & $30.91^{c}$ & $5.77^{\mathrm{a}}$ & $0.41^{\mathrm{c}}$ & $5.82^{\mathrm{c}}$ & $91.63^{d}$ \\
\hline 4 & $30.21^{d}$ & $3.04^{\mathrm{b}}$ & $-1.07^{\mathrm{d}}$ & $3.27^{d}$ & $308.34^{\mathrm{a}}$ \\
\hline 5 & $28.01^{\mathrm{e}}$ & $1.29^{\mathrm{c}}$ & $-1.67^{\mathrm{e}}$ & $2.14^{\mathrm{e}}$ & $331.82^{\mathrm{a}}$ \\
\hline Tukey $_{0, \mathrm{C}}$ & 0.54 & 0.37 & 0.41 & 0.47 & 34.29 \\
\hline
\end{tabular}

Letras distintas indican que el valor es significativamente diferente entre los estados de madurez.

El valor inicial de $\mathrm{a}^{*}$ en los frutos de color verde $100 \%$ (estado de madurez 1) fue de $-5,68$ aumentando 11 unidades en el estado $3\left(a^{*}=5,77\right)$ alcanzando al final de la maduración un valor de 1,29 (estado de madurez 5). Los cambios ocurridos en $a^{*}$ se deben a que cuando el color tiende a rojo los valores de $a^{*}$ aumentan en sentido positivo de acuerdo al plano del espacio de color $L^{*} a^{*} b^{*}$ y por el contrario, si el color tiende a verde los valores de $a^{*}$ aumentan en sentido negativo (Kónica Minolta, 2012; Sinnecker, et. al., 2002). Valores similares a los obtenidos para los frutos de mortiño fueron reportados por Sinnecker, Gomes, Areas, \& Marquez (2002) en semillas de soya y por Zheng, et. al. (2003) para arándano azul.

En cuanto a los valores de $b^{*}$ se obtuvo un valor inicial de 10 (mortiño verde $100 \%$ ) mientras que en el estado 5 (madurez hortícola) se obtuvo un valor -1,67. Estos cambios se producirían debido a que cuando el color tiende a azul la medida de $b^{*}$ aumentará en sentido negativo de acuerdo al 
plano del espacio de color $\mathrm{L}^{*} \mathrm{a}^{*} \mathrm{~b}^{*}$, y si el color tiende a amarillo, la medida de $\mathrm{b}^{*}$ aumentará en sentido positivo (Kónica Minolta, 2012).

A lo largo de la maduración el valor de Croma $\left(\mathrm{C}^{*}\right)$ disminuyó gradualmente, según se observa en la tabla 2. En el estado de madurez 1 se obtuvo un valor de $C^{*}=11,84$ mientras que en el estado de madurez 5 se encontró un valor de 2,14. La saturación del color disminuyó conforme aumentó el estado de madurez de la fruta; lo que indica que mientras el color tiende a verde, se presenta una saturación mayor y mientras el color tiende a negro, la saturación será menor. Resultados similares a los obtenidos en la presente investigación fueron reportados por Zheng et. al. (2003) en arándano azul; mientras que los valores del ángulo Hue se incrementaron durante la maduración del mortiño de $155^{\circ}$ (estado madurez 1) a $331^{\circ}$ (estado madurez 5). Resultados similares han sido reportados en manzana (Greer, 2013) y arándano (Zheng, et. al., 2003).

Tabla 3. Diferencia de color $(\Delta \mathrm{E})$ durante la maduración del mortiño (Vaccinium floribundum)

\begin{tabular}{ccccc}
\hline Estado de Madurez & $\mathbf{2}$ & $\mathbf{3}$ & $\mathbf{4}$ & $\mathbf{5}$ \\
\hline $\mathbf{1}$ & 21,551 & 135,507 & 129,219 & 140,510 \\
$\mathbf{2}$ & -- & 54,241 & 55,474 & 72,128 \\
$\mathbf{3}$ & -- & -- & 5,164 & 16,575 \\
$\mathbf{4}$ & -- & -- & -- & 4,127 \\
\hline
\end{tabular}

Al analizar la diferencia de color $(\Delta \mathrm{E})$, según se observa en la Tabla 3, se encontró que entre los estados de madurez 1 y 2 (mortiño verde y verde-rosado, respectivamente) la diferencia fue de 21,6 unidades; entre los estados 2 y 3 (mortiño rosado) el $\Delta \mathrm{E}$ fue de 54,2 unidades. Al avanzar el proceso de maduración de la fruta esta diferencia disminuyó a 5,2 unidades entre el estado 3 (madurez fisiológica) y 4 (mortiño negro-rosado). Cuando el mortiño pasó del estado 4 al 5 (mortiño negro) y alcanzó la madurez comercial, la diferencia de color fue de 4,1 unidades. Se pudo observar que el cambio de color en los estados 1-2 y 2-3 es mayor en comparación con el cambio entre los estados 3-4 y 4-5, resultados similares fueron reportados por Qudsieh et. al., (2002) indicando que durante el proceso de maduración esta diferencia disminuye debido a que la fruta alcanza la madurez y además existe concentración de sustancias que aportan color.

\subsection{Contenido de antocianinas}

El contenido de antocianinas en los diferentes estados de maduración de mortiño se indica en la tabla 4. En el estado de madurez 1 la concentración de antocianinas fue de $41 \mathrm{mg} / \mathrm{Kg}$ cianidina-3glucósido (mortiño verde) similares a los valores reportados por Kalt, et. al. (2003) en diferentes variedades de arándano azul. Cuando los frutos de mortiño alcanzaron el estado de madurez 2 (mortiño verde-rosado) se produjo un incremento en relación 1:10 teniendo una concentración de antocianinas 415 mg/Kg cianidina-3-glucósido; Kalt, et. al. (2003) indica que el contenido de 
antocianinas para arándanos de color rosado se encuentra entre 75 a $450 \mathrm{mg} / \mathrm{Kg}$ cianidina-3glucósido según la variedad, siendo estos valores similares a los del mortiño.

Tabla 4. Contenido de antocianinas en los diferentes estados de madurez del mortiño (Vaccinium

\begin{tabular}{cc}
\hline $\begin{array}{c}\text { Estado de } \\
\text { Madurez }\end{array}$ & $\begin{array}{c}\text { Antocianinas } \\
\text { (mg/Kg cianidina-3-glucósido) }\end{array}$ \\
\hline 1 & $40,97^{\mathrm{e}}$ \\
2 & $415,27^{\mathrm{d}}$ \\
3 & $1295,9^{\mathrm{c}}$ \\
4 & $3287,39^{\mathrm{b}}$ \\
5 & $13830^{\mathrm{a}}$ \\
\hline Letras distintas indican que el valor es significativamente diferente entre los & estados de madurez.
\end{tabular}

Cuando el fruto alcanza la madurez fisiológica (estado 3), se encontró una concentración de antocianinas de $1295 \mathrm{mg} / \mathrm{Kg}$ cianidina-3-glucósido, mientras que cuando los frutos de mortiño alcanzar el estado de madurez 4 (negro-rosado) la concentración sufrió un incremento casi dos veces mayor alcanzando valores de $3280 \mathrm{mg} / \mathrm{Kg}$ cianidina-3-glucósido; valores similares fueron reportados por Wang, et. al. (2009) para arándano azul. En el estado de madurez 5 (madurez hortícola) los frutos de mortiño presentaron una coloración negruzca y la concentración de antocianinas se incrementó hasta 13800 mg/Kg cianidina-3-glucósido, siendo mayor al contenido de antocianinas en diferentes variedades de arándano azul en los que se han encontrado valores entre 7400 y 9200 mg/Kg cianidina-3-glucósido (Kalt, et. al., 2003).

La concentración de antocianinas se incrementa durante la maduración en todas las especies de Vaccinium. Este aumento se produciría debido a que las bayas en las primeras etapas de madurez contienen clorofilas (color verde), las cuales se degradan progresivamente siendo sustituidas por las antocianinas, las mismas que se manifiestan con la coloración característica roja, morada o negra en el caso del mortiño que se complementa por una serie de cambios relacionados con el desarrollo de compuestos tales como taninos y otros ácidos orgánicos, dando como resultado colores más oscuros y variados (Kalt, et. al., 1999; Qudsieh, et. al., 2002; Coleto, 1994).

\subsection{Cuantificación de clorofila}

Se determinó el contenido de clorofila $\alpha, \beta$ y total en los diferentes estados de madurez del mortiño (tabla 5). El mayor contenido de estos compuestos se encontró en estado de madurez 1 y los menores valores se encontraron en los estados de madurez (4 y 5). Los valores de clorofila $\alpha$ y $\beta$ que se obtuvieron en la presente investigación, para los frutos de mortiño, son mayores que los reportados por Brotons, Manera, Conesa, \& Porras, (2013) en brócoli (Brassica oleracea) y por 
el contrario, son menores a los presentados por Burns, Fraser, \& Bramley (2003) en lechuga y pimiento, esta diferencia indica que existe variación del contenido de clorofila en cada vegetal.

Tabla 5. Contenido de clorofila $\alpha, \beta$ y totales en el mortiño (Vaccinium floribundum)

\begin{tabular}{cccc}
\hline $\begin{array}{c}\text { Estado de } \\
\text { Madurez }\end{array}$ & $\begin{array}{c}\text { Clorofila } \boldsymbol{\alpha} \\
(\mathbf{m g} / \mathbf{g})\end{array}$ & $\begin{array}{c}\text { Clorofila } \boldsymbol{\beta} \\
(\mathbf{~ m g} / \mathbf{g})\end{array}$ & $\begin{array}{c}\text { Clorofila Total } \\
(\mathbf{m g} / \mathbf{g})\end{array}$ \\
\hline 1 & 0,045 & 0,030 & $0,075^{\mathrm{a}}$ \\
2 & 0,035 & 0,018 & $0,053^{\mathrm{b}}$ \\
3 & 0,019 & 0,014 & $0,032^{\mathrm{c}}$ \\
4 & 0,013 & 0,010 & $0,023^{\mathrm{d}}$ \\
5 & 0,010 & 0,009 & $0,019^{\mathrm{e}}$ \\
\hline
\end{tabular}

Letras distintas indican que el valor es significativamente diferente entre los estados de madurez.

El contenido de clorofila disminuye durante la maduración de los frutos. La cantidad de clorofilas totales contenidas en el mortiño antes de empezar el proceso de maduración fue de 0,075 mg/g (tabla 5). La concentración de clorofila varía según las especies y será mayor en aquellas que presentan color verde a lo largo del proceso de maduración por ejemplo: brócoli $(0,0191 \mathrm{mg} / \mathrm{g})$, limón verde $(0,1590 \mathrm{mg} / \mathrm{g})$ y melón verde $(0,20 \mathrm{mg} / \mathrm{g})$ (Burns, Fraser, \& Bramley, 2003; Srilaong, Aiamla-or, Soontornwat, Shigyo, \& Yamauch, 2011; Tadmor, et. al., 2010).

Cuando los frutos de mortiño inician el proceso de maduración toman coloración rosada en ciertas partes del fruto (estado de madurez 2), manteniendo varias zonas con color verde. La cantidad de clorofila en esta etapa disminuyó a $0,053 \mathrm{mg} / \mathrm{g}$ y cuando la fruta alcanzó la madurez fisiológica (estado de madurez 3-color rosado) el contenido de clorofila alcanzó valores de 0,032 mg/g.

En el estado de madurez 5 (madurez hortícola) los frutos presentan una coloración negruzca, la concentración de clorofilas disminuyó a 0,019 mg/g. Zude (2003) reportó valores similares en manzana; mientras que se han reportado valores ligeramente mayores en remolacha (Arnon; 1949) y en frutos de coloración negruzca como el pimiento jalapeño (Moreno et. al.,2010).

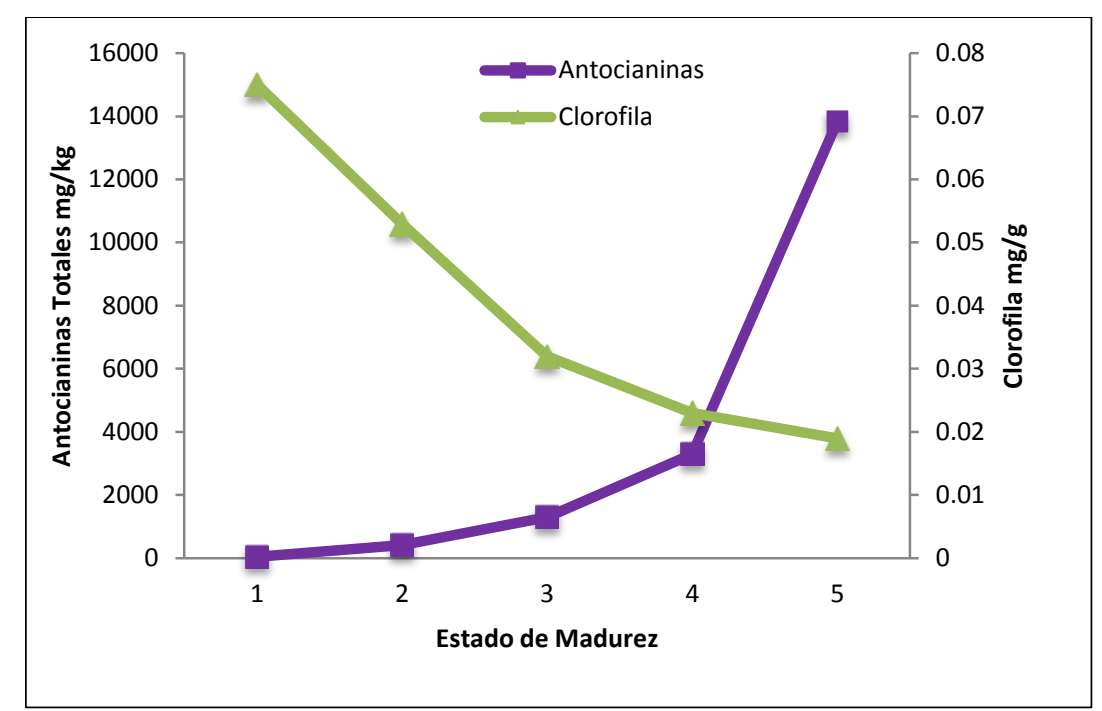

Figura 2. Relación del contenido de antocianinas y clorofilas por estado de madurez 
El color se toma como una característica de calidad sensorial y valor nutricional (Brotons et al, 2013). Se analizó la relación entre el contenido de antocianinas y clorofila en los diferentes estados de maduración de mortiño (figura 2); en el estado de madurez 1 presentaron una alta concentración de clorofilas en comparación a los estados 4 y 5 , es decir que, mientras avanza el proceso de maduración el contenido de clorofilas disminuye y ocurre lo contrario con las antocianinas, las cuales se encuentran en baja cantidad en las primeras etapas de maduración (estados de madurez 1 y 2) e incrementan gradualmente hasta llegar a una alta concentración en los estados de madurez 3,4 y 5 . Siendo el mortiño un producto rico en antocianinas, su determinación en diferentes estadíos de maduración permitiría el aprovechamiento del fruto para la extracción de compuestos bioactivos.

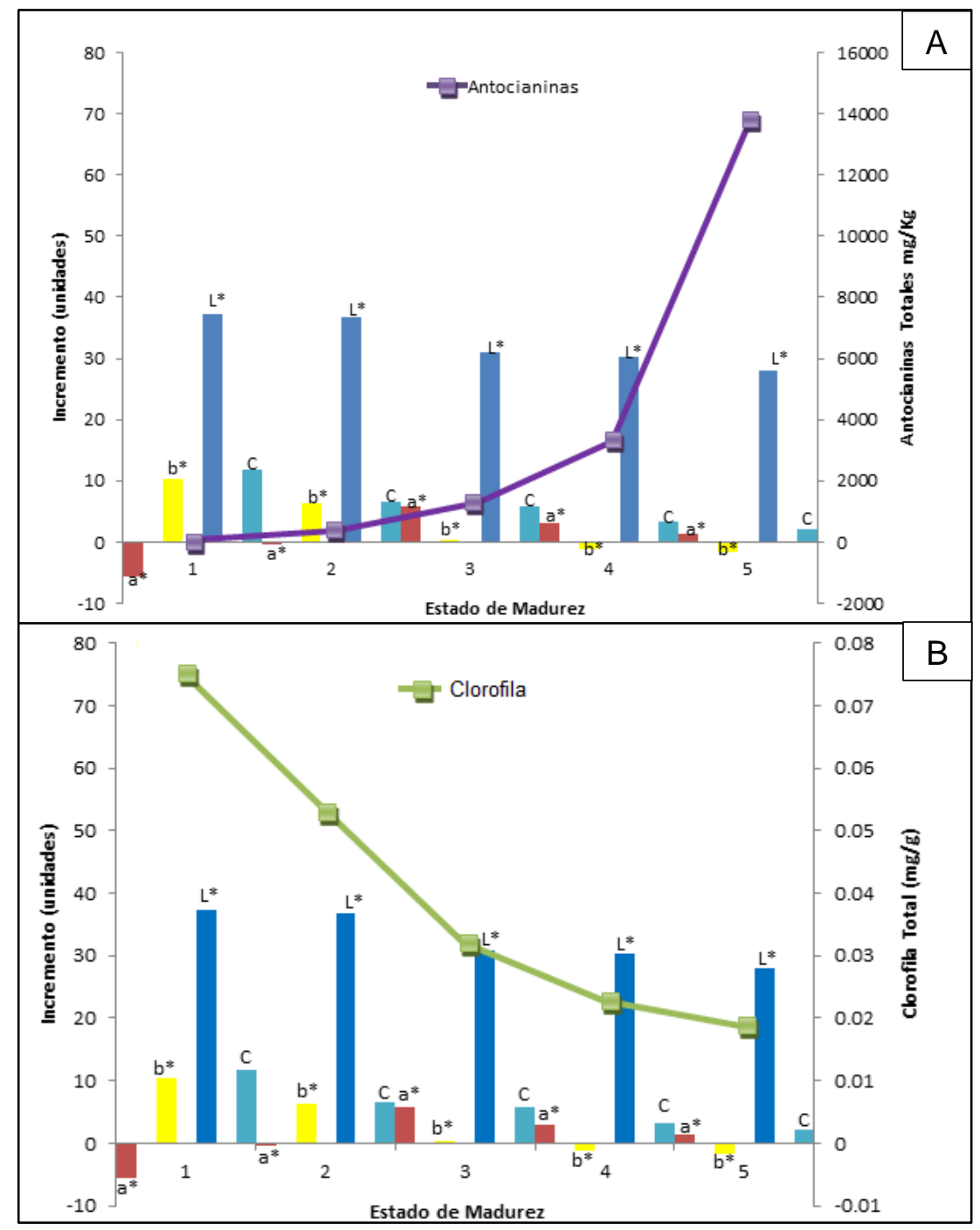

Figura 3. Relación de las medidas de $a^{*}, b^{*}$, Luminosidad y Croma con el contenido de $(A)$ Antocianinas Totales y (B) Clorofila total en mortiño (Vaccinium floribundum)

En la figura 3A se puede observar la relación entre el contenido de antocianinas y los parámetros de color $L, a^{*}, b^{*}$ y $C^{*}$. Cuando la fruta presenta colores con altos valores de Luminosidad y 
Saturación, y valores negativos para la medida de $a^{*}$ y positivos para la medida de b*, la concentración de antocianinas es baja. A medida que aumenta el contenido de antocianinas, los valores de luminosidad y saturación son bajos, y se encuentran acompañados de valores positivos de $a^{*}$ y negativos de $b^{*}$. En contraste con lo que ocurre con el contenido de clorofila (figura 3B), cuanto éste es mayor la fruta presenta medidas altas de Luminosidad y Saturación, y valores negativos para $a^{*}$ y positivos para $b^{*} ; y$, por lo contrario cuando la concentración de clorofila disminuye, el mortiño presenta medidas de luminosidad y saturación bajos, acompañado de valores positivos para $a^{*}$ y negativos para $b^{*}$.

\section{Conclusiones y Recomendaciones}

La medida de luminosidad (L) y la saturación (Croma) descienden conforme la fruta aumenta su estado de madurez, mientras que los valores del ángulo Hue fueron los correspondientes al cuadrante del plano de color que caracteriza cada estado de madurez. Además, se obtuvieron valores positivos de $a^{*}$ y negativos de $b^{*}$ para los estados de madurez que tienden hacia el color rojo y azul respectivamente (estados 3,4 y 5); y valores negativos de $a^{*}$ y positivos de $b^{*}$ para los estados de madurez que tienden al color verde y amarillo, respectivamente (estados 1 y 2 ).

A medida que avanzó el proceso de maduración se produjo una reducción del $70 \%$ del contenido de clorofilas entre los estados de madurez 1 (mortiño color verde) y 5 (mortiño color verde). Esta diferencia de concentración está directamente relacionada con el color, que es una característica física que indica qué tipo de compuestos están contenidos mayoritariamente en una fruta, de forma que se establezca una relación directa entre el color y el contenido de compuestos coloreados como clorofila y antocianinas.

Por otro lado, los resultados encontrados permiten el planteamiento de nuevos temas de investigación como la relación entre la variación de color que sufre la fruta durante el proceso de maduración y el contenido de compuestos antioxidantes presentes en el mortiño, de modo que se pueda aprovechar los frutos en diferentes estados de madurez constituyendo una fuente de compuestos bioactivos dada la importancia que tienen estos compuestos y su implicación en la salud humana. Además, la promoción del consumo de productos con ingredientes funcionales como el mortiño aumentaría su demanda en mercados nacionales así como generar fuentes agroindustriales con efectos socio económicos en las zonas de producción.

\section{Bibliografía}

Aguilera, M., Reza, M., Chew, R., \& Meza, J. (2011). Propiedades Funcionales de las Antocianinas. Revista Biotecnia-Revistade Ciencias Biológicas y de la Salud de la Universidad de Sonora, 16. 
Allinger, Cava, De Jongh, Jhonson, Lebel, \& Stevens. (1984). Química Orgánica. Barcelona: Worth Publishers, Inc.

Andrade, J. (2012). Estudio de la capacidad antioxidante total y contenido de compuestos antioxidantes en mortiño (Vaccinium Floribundum) tratado con luz UV-C. Tesis de Ingeniería en Alimentos. Universidad Tecnológica Equinoccial. Quito, Pichincha, Ecuador.

Arnon, D. (1949). Coper Enzymes in isolated choloroplast. Polyphenoloxidase in beta vulgaris. Plant Physol, 1-15.

Artigas, J., Capilla, P., \& Pujol, J. (2002). Tecnología del Color. Valencia: Maite Simon.

Badui, S. (1981). Química de los Alimentos. México: Alhambra Mexicana.

Barreiro, J., \& Sandoval, A. (2006). Operaciones de Conservación de Alimentos por bajas temperaturas. Caracas: Equinoccio.

Beas, e. A. (2011).

Beas, R., Loarca, G., Guzmán, S., Rodríguez, M., Vasco, N., \& Guevara, F. (2011). Potencial nutracéutico de componentes bioactivos presentes en huitlacoche de la zona centro de México. Revista Mexicana de Ciencias Farmacéuticas, 36-44.

Brotons, J. M., Manera, J., Conesa, A., \& Porras, I. (2013). A fuzzy approach to the loss of green color in lemon (Citrus lemon L. Burns. f.) rind during ripening. Compurers and Electronics in Agriculture, 222-232.

Burns, J., Fraser, P., \& Bramley, P. M. (2003). Identification and quantification of carotenoids, tocophenols and chlorophylls in commonly consumed fruits and vegetable. Science Direct, 939-947.

Canjura, F. J., \& Schwartz, S. J. (1991). Saparation of chlorophyll compounds and their polar derivatives by high-performance liquid chromatography. J. Agric. Food Chem, 1102-1105.

Cogua, J., Orozco, M., \& Chaparro, Á. (2002). Pigmentos Fotosintéticos. Fisiología Vegetal. Bogotá.

Coleto, J. M. (1994). Crecimiento y Desarrollo de las especies frutales. Madrid: Grupo MundiPrensa.

Comité Ambiental de Cotopaxi. (2004). Iniciativa para el Uso de los Recursos Naturales de Cotopaxi. Serie Comité Ambiental de Cotopaxi 1, (pág. 54). Latacunga. 
Dávila, J., Andrés, C., Stalin, R., \& Francisco, B. (2010). Ingredientes Funcionales de Plantas Ecuatorianas. Revista Politécnica, 51-59.

Ege, S. (2000). Química Orgánica Estructura y Reactividad. Barcelona: Reverté S.A.

Estrella, E. (1988). El pan de América etnohistoria de los alimentos aborígenes del Ecuador. Quito: Abya-yala.

Fennema, O. (2000). Química de los Alimentos. Zaragoza: ACRIBIA S.A.

Fuentes, V. (2008). Estudio del Mortiño y propuesta gastronómica aplicada a un recetario. Tesis de gastronomía. Universidad Tecnológica Equinoccial. Quito, Pichincha, Ecuador.

Gonzalez, I. (2010). Caracterización química del color de diferentes variedades de guayaba (Psidium guajava L.) colombiana. Tesis de Química. Universidad Nacional de Colombia. Bogotá, Colombia.

Greer, D. (2013). Non-destructive chorophyll fluorescence and colour measurements of Braeburn and Royal Gala apple (Malus domestica) fruit development throughout the growing season. New Journal of Crop and Horticultural Science, 413-421.

Hernandez, L., \& González, C. (2002). Introducción al Análisis Instrumental. Barcelona: Ariel S.A. Hunter Lab. (2001). Principios básicos de medida y percepción de color. Recuperado el 3 de Enero de 2013, de www.hunterlab.com

Jimenez, V. (2004). Determinación de métodos para producción de mortiño (Vaccinum Floribumdun), con fines de propagación y producción comercial. Quito, Pichincha, Ecuador. Kader, A. A., Adaskaveg, J. E., Crisosto, C. H., Arpaia, M. L., Edwards, D. E., Barret, D. M., y otros. (2007). Tecnología Postcosecha de Cultivos Hortofrutículas. California: UC Peer Reviewed.

Kalt, W., Forney, C., Martin, A., \& Prior, R. (1999). Antioxidant Capacity, Vitmin C, Phenolics, and Anthocyanins after Fresh Storage of Small Fruits. Journal of Agricultural and Food Chemestry, 4638-4644.

Kalt, W., Lawand, C., Ryan, D., McDonald, J., Donner, H., \& Forney, C. (2003). Oxygen Radical Absorbing Capacity, Anthocyanin and Phenolic Content of Highbush Blueberries (Vaccinium Corymbosum L.) during Ripening and Storage. Journal of Agricultural and Food Chemestry, 917-923. 
Konica Minolta. (2012). Comunicación Precisa de los colores. Recuperado el 30 de Octubre de 2012, de http://www2.konicaminolta.eu/eu/Measuring/pcc/es/index.html

Kónica Minolta. (2012). Comunicación Precisa de los colores. Recuperado el 30 de Octubre de 2012, de http://www2.konicaminolta.eu/eu/Measuring/pcc/es/index.html

La Cie Group. (2006). Libro blanco de la gestión del color 3 Espacios de color y conversión de colores. New York: Evergreen Parkway.

Lide, D. (2004). Handbook of Chemestry and Physics. New York: CRC PRESS.

Lincoln, T., \& Zeiger, E. (2002). Fisiología Vegetal. Jaume: Sinauer Associates.

Moreno, S., Guerra, J. A., Cárdenas, M. L., Nuñez, M. A., Gámez, H., \& Villareal, J. A. (28 de Mayo de 2010). Determinación de Carotenoides y Clorofila en frutos de cuatro variedades de Chile (Capsicum sp). Guanajuato, Mexico.

Muñoz, V. (2004). Determinación de Métodos para producción de mortiño (Vaccinum Floribundum Kunth), con fines de propagación y producción comercial. Tesis de Ingeniería en Agroempresas. Universidad San Francisco de Quito. Quito, Pichincha, Ecuador.

Natakani, H. Y., Barber, J., \& Forrester, J. A. (1979). Surface charges on chloroplast membranes as studies by particle electrophoresis. Biochim. Biophys, 215-225.

Nielsen, S. (2009). Análisis de los Alimentos. Zaragoza: ACRIBIA, S.A.

Noboa, V. (2010). Efecto de seis tipos de sustratos y tres dosis de ácido alfa naftalenacético en la propagación vegetativa de mortiño (Vaccinum Floribundum Kunth). Tesis de Ingeniería Forestal. Escuela Superior Politécnica de Chimborazo. Riobamba, Ecuador.

Pascual, S., \& Sánchez, M. (2007). "Anthocyanins: from plant to health". Phytochemestry review.

Patiño, V. (2002). Historia y Dispersión de los Frutales Nativos del Neotrópico. Cali.

Patzett, E. (1996). Flora del Ecuador. Quito, Pichincha, Ecuador.

Pérez, S., \& Valdiviezo, C. (26 de Enero de 2007). Colección y caracterización morfológica In Situ del mortiño (Vaccinum Floribundum Kunt) en la sierra norte del Ecuador. Tesis de Ingeniería Agropecuaria. Escuela Politécnica del Ejercito. Sangolquí, Pichincha, Ecuador.

Pokorny, J., Yanishlieva, N., \& Gordon, M. (2001). Antioxidantes de los Alimentos Aplicaciones Prácticas. Zaragoza: ACRIBIA S.A.

Popeneo, H., King, S., León, J., \& Sumar, L. (1989). Lost Crops of the the Incas. Washington D.C. 
Qudsieh, H., Yusof, S., Osman, A., \& Rahman, R. (2002). Effect of maturity on chlorophyll, tannin, color and polyphenol oxidase (PPO) activity of sugarcane juice (Saccharum officinarum Var. Yellow Cane). Agricultural and Food Chemestry, 1615-1618.

Rivera, J., Vasquez, F., Ayala, J., \& Gonzalez, G. (Enero de 2005). Efecto del corte y la temperatura de almacenamiento en la calidad de Papaya fresca cortada (Carica Papaya L. CV. "maradol"). Revista Iberoamericana de Tecnología Postcosecha, 83-94.

Sinha, N., Sidhu, J., Barta, J., Wu, J., \& Cano, P. (2012). Handbook of fruits and fruit processing. lowa: Wiley-Blackwell.

Sinnecker, P., Gomes, S., Areas, J., \& Marquez, U. (2002). Relationship between color (Instrumental and Visual) and Chorophyll Contents in Soybean Seeds during Ripening. Journal of Agricultural and Food Chemistry, 3961-3966.

Skoog, D., West, D., Holler, J., \& Crouch, S. (2001). Química Analítica. México: Mc GRAW-HILL.

Sociedad Latinoamericana de Nutrición. (1999). Archivos Latinoamericanos de Nutrición. Texas: La Sociedad.

Srilaong, V., Aiamla-or, S., Soontornwat, A., Shigyo, M., \& Yamauchi, N. (2011). UV-B irradiatio retards chlorophyll degradation in lime (Citrus lactifolia) fruit. Postharvest Biology and Technology, 110-112.

Strain, H. H., Thomas, M. F., \& Katz, J. J. (1963). Spectral absorption properties of ordinary and fully deuteriated chloropylls a and b. Biochim. Biophys, 306-311.

Tadmor, Y., Burger, J., Yaakov, I., Feder, A., Libhaber, S., Portnoy, V., y otros. (2010). Genetics of Flavonoid, Carotenoid and Chlorophyll Pigments in Melon Fruit Rinds. Journal of Agricultural and Food Chemestry, 10722-10728.

Tupuna, D. (Septiembre de 2012). Obtención de jugo clarificado concentrado de mortiño (Vaccinium Floribundum) mediante el uso de tecnología de membranas. Tesis de Ingeniería Química y Agroindustria. Escuela Politécnica Nacional. Quito, Pichincha, Ecuador.

Vargas, W. (2002). Guía Ilustrada de las Plantas de las Montañas del Quindío y los Andes Centrales. Caldas: Universidad de Caldas. 
Vasco, C. (2009). Phenolic Compounds in Ecuadorian Fruits. Tesis Doctoral. Swedish University of Agricultural Sciences. Uppsala, Sweden.

Vasco, C., Avila, J., Ruales, J., Svanberg, U., \& Kamal-Eldin, A. (2009). Physical and chemical characteristics of golden-yellow and purple-red varieties of tamatillo fruit (Solanum betaceum Cav.). International Journal of Food Sciences and Nutrition, S1-S11.

Vasco, C., Riihinen, K., Ruales, J., \& Kamal-Eldin, A. (2009). Chemical Composition and Phenolic Compound Profile of Mortiño (Vaccinium floribundum Kunth). Journal of Agricultural and Food Chemestry, 1204-1212.

Viña, S., Mugridge, A., Olivera, D., Chaves, A., \& Mascheroni, R. (2008). Características y Usos Potenciales de Frutos de Okra de acuerdo al Cultivar de Origen. La Plata, Argentina.

Viñas, I., Recansens, I., Usall, J., \& Graell, J. (2013). Poscosecha de per, manzana y melocotón. Mundi Prensa Libros.

Wang, C., Chen, C.-T., \& Wang, S. (2009). Changes of flavonoid content and antioxidant capacity in the blueberries after illumination with UV-C. Food Chemistry, 426-431.

Yang, X., Song, J., Fillmore, S., Pang, X., \& Zhang, Z. (2011). Effect of High temperature on color, chorophyll fluorencece and volatile biosynthesis in green-ripe banana fruit. Postharvest Biology and Technology, 246-257.

Zheng, Y., Chien, W., Shiow, W., \& Wei, Z. (2003). Effect of High Oxygen Atmospheres on Blueberry Phenolics, Anthocyanins, and Antioxidant Capacity. Journal of Agricultural and Food Chemestry, 53.

Zude, M. (2003). Comparison of indices and multivariate models to non-destructively predict the fruit chlorophyll by means of visible spectrometry in apple fruit. Science Direct, 119-126. 